\title{
Indoor positioning system for movement path analysis in healthcare institutions
}

\author{
Stefan Grönroos, $\mathrm{PhD}^{1}$, Laura-Maria Peltonen, $\mathrm{MNSc}^{2}$, Valentin Soloviev, $\mathrm{Mr}^{1}$, Johan Lilius, PhD, professor ${ }^{1}$, Sanna
} Salanterä, PhD, professor ${ }^{2}$

${ }^{1}$ Åbo Akademi University, Turku, Finland; ${ }^{2}$ Nursing Science, University of Turku and Turku University Hospital Turku, Finland

Johan Lilius, Åbo Akademi University, Turku, FINLAND. Email: johan.lilius@abo.fi

\begin{abstract}
In this paper, we describe an indoor positioning system designed to provide data on the movement patterns of hospital personnel. The data collection is ongoing and part of a larger study project, which aims to evaluate the impact of an information system implemented in a hospital setting. The indoor positioning system was designed to be non-intrusive and straightforward to deploy in multiple hospitals, while requiring minimal existing infrastructure. To this end, the system is based on battery-powered Bluetooth beacons, and mobile phones measuring the signal strength of the beacons to position their bearers. This paper describes the design and implementation of the system. We analyze the positioning accuracy of the system in order to evaluate its fitness for purpose. Based on the results, the system is suitable for short-term deployments due to its low cost and ease of installation.
\end{abstract}

Keywords: indoor positioning, navigation, bluetooth low energy, hospitals

\section{Introduction}

Tracking technology has a variability of applications in the health care setting and research on this technology is on the increase. The technology often utilizes wearable devices such as smartphones (see e.g. [1-3]) or smart-watches [4], and it may also be implemented in garments (see e.g. $[5,6]$ ). The cost and effort needed to obtain and deploy a fancy system may however become arduous for research purposes. Therefore, easy to use and low cost solutions are also needed.

This paper describes the design of an indoor positioning system used as one data collection method in a larger study with the aim to explain the impact of an information system on the patients' care processes and the shift leaders' work in a hospital setting. In this larger study, the indoor positioning data is collected to describe the differences in the movement patterns of the professionals responsible for the daily coordination of care during a specific shift, including nurses and physicians. This larger study is conducted in the emergency departments of three Finnish hospitals and the data is collected one month at a time for five months in total during 2015-2016. All data is collected anonymously to protect the participants. The nurses' and the physicians' movement patterns are explored separately in each participating unit. The interest lies on the movement patterns of these professionals during each data collection month in total and not on the movement of any specific individual. The movement patterns are compared before and after the implementation of the information system. 
This paper presents the indoor positioning system and its design together with the validation in a controlled environment (the Agora building in Turku and a pilot test in one hospital unit). The goal is to evaluate the performance and suitability of a simple and low cost indoor positioning system for the larger experiment being conducted in the hospitals. A separate paper wil later present the findings on the movement patterns in the hospitals.

The paper is laid out as follows. In the Materials and Methods section, we describe the requirements and design choices made for the system; the components of the indoor positioning system; and the measurements for validating the system. The analyses of the measurements are presented in the Results section, which is followed by the Discussion and ethical considerations.

\section{Material and methods}

\section{System requirements and background}

The system was to be deployed by untrained personnel, so from the outset it had to be designed to be simple. Overall the most important requirements for the system were:

- room-level accuracy preferred

- simplicity, and thus short development cycle

- minimal supporting infrastructure should be necessary

- quick and easy deployment and disassembly, to be performed by personnel not familiar with the system

- fairly inexpensive, since the system would only be used for one series of studies

- safety and confidentiality issues should be acknowledged.

There are many different approaches to indoor positioning, many of which use radio signals for positioning. In addition, to radio-based positioning techniques there are also techniques based on using sound, computer vision, and detecting variations in magnetic fields for example [7].
Most technologies require some supporting infrastructure - i.e. radio transmitters, cameras, loudspeakers etc. - to work. An example of an infrastructure- less technology is using the magnetometer (compass) found in many mobile phones to build a map of magnetic field variations in a building [8]. This requires building a map of the building first, however, which decreases the simplicity of deployment by untrained personnel.

Since $\mathrm{Wi}-\mathrm{Fi}$ is commonly found in many buildings, navigation using received signal strength indication (RSSI) from different Wi-Fi access points has been a rather popular option. The density and placement of $\mathrm{Wi}-\mathrm{Fi}$ base stations in the building will have a major impact on performance in such a system, however. Relying on existing Wi-Fi infrastructure would have introduced too much risk, and would have required obtaining maps of base station placement in each facility in our case. A number of commercial WiFi based locationing systems exist (eg. Walkbase ${ }^{1}$ ) however they are built for specific purpouses, and cannot readily be translated into a hospital environment. Recently, Bluetooth Low Energy (BLE) [9] beacons have been introduced, using simple protocols such as Apple's iBeacon protocol to broadcast a unique identifier to nearby BLE-enabled devices. Depending on how often, and at which signal strength the identifier is broadcast, these beacons can often run on a coin- cell battery for over a year. The beacons are also relatively low cost, at a typical price point of USD 10-30 per beacon. We have not found any similar system in the literature, that would satisfy our requirements on ease of setup and of accuracy.

A device supporting BLE can thus receive the broadcast identifiers from beacons, as well as give an RSSI. The RSSI can be used to roughly estimate the receiver's distance from the beacon $[10,11]$. We decided to use BLE beacons for our indoor positioning system as it fulfills the requirements of the system quite well. Upfront development is quite simple, as we were able to develop a mobile application that listens for BLE beacons in range, and logs their ID and RSSI to a database.

\footnotetext{
${ }^{1}$ http://www.walkbase.com/
} 
Due to the low cost of BLE beacons, we would be able to place one beacon in each room in the parts of the building we wanted to cover. We would also place some beacons outside rooms; in hallways, near building entrances etc.

The dense placement of beacons means that even simple algorithms - such as the assumption that the receiver is located at the position of the beacon with the strongest RSSI - will give fairly accurate results. The logging of all beacon observations to the database, however, also enables the development of more sophisticated algorithms, taking into account more distant beacons, to run over the collected data.

Installation of the system is straightforward for untrained personnel, since it only involves placing a beacon in each room of interest, and noting the ID and position of each beacon on a map. Use of the system ideally only involves carrying a mobile phone in the pocket, and making sure to charge the phone regularly.

From a safety standpoint, the beacons do transmit at 2.4 GHz similarly to $\mathrm{Wi}-\mathrm{Fi}$, and the system might thus be unsuitable for use in certain locations, where the use of cell phones is prohibited. From a privacy perspective, the beacons only emit signals and do not collect any information. In order to be tracked, one has to carry a phone running the tracking application. We discuss the ethical issues in more detail in section Ethical considerations.

\section{Experimental setup}

The indoor positioning system consists of BLE beacons deployed at locations of interest. Each person to be tracked carries an Android smartphone equipped with a Bluetooth receiver.

The Android application includes a local Couchbase Lite database, into which measurement results are stored. If Internet connectivity is present, the local database content is also transferred to a master Couchbase database running on a server. The Android application uses the Android Beacon Library4 to scan for BLE beacons roughly every 30 seconds. For each scan period, the following data is stored in the database:

1. The time of the scan

2. An ID unique to the phone

3. The phone's battery level

4. A list of each beacon found with the following information:

- The unique beacon ID

- $\mathrm{RSSI}$ in $\mathrm{dBm}$, an estimate of the distance to the beacon in meters as calculated by the Android Beacon Library

- A value for the transmission power of the beacon in $\mathrm{dBm}$ (for calibration)

In the database, we also store the map coordinates of each beacon. The knowledge of each beacon's location combined with the measured signal strengths from each beacon allows us to estimate the location of the smartphone for each scan period.

\section{Measurements}

In order to evaluate the accuracy of the indoor positioning system, we installed beacons on one floor of Agora, a building on a university campus in Turku, Finland. The beacons were placed so that one beacon was present in each room, where measurements were made. Beacons were also placed in hallways and open spaces. The beacons used were the iBKS 105 and 105A models from Accent Systems. These beacons were also deployed in hospitals.

Measurements were performed in 8 different locations. Two phones were used at each location. The phones used were the same Motorola Moto-E devices that were also used for measurements in hospitals. One phone was laid on a table, and the other phone was placed in the pocket of a person sitting at each location. We measured for roughly 10-20 minutes at each location. The locations measured are described in table 1.

All rooms contained at least one beacon, except for SR2, a small "quiet-room" inside a larger open-plan office. A large coffee-room/sitting area contained four beacons placed in each corner of the area. 
The location of the phone, based on measured RSSI for each beacon within range, was estimated using two different algorithms. The first algorithm is the simplest.
Here we estimate the position of the phone to be the position of the beacon with the strongest RSSI.

Table 1. Measurement locations.

\begin{tabular}{lll}
\hline Location & Description & Beacon in room? \\
\hline MR1 & Meeting room & Yes \\
MR2 & Meeting room & Yes \\
CR (mid) & Middle of large coffee-room/sitting area & Yes (4 beacons) \\
CR (N) & Upper part (on map) of coffee-room & Yes (4 beacons) \\
CR (NE) & Upper-right (on map) part of coffee-room & Yes (4 beacons) \\
SR1 & Small room inside an open-plan office space & Yes \\
SR2 & Small room inside an open-plan office space & No \\
SR3 & Small room near MR2 & Yes \\
\hline
\end{tabular}

The second position-estimation algorithm uses all beacons within range to estimate the position. The distance $d$ to a beacon is estimated according to the following formula in the Android Beacon Library [5]:

$$
d=C_{1} \times\left(\frac{P_{r s s i}}{P_{t x}}\right)^{C_{2}}+C_{3}
$$

where $C_{1}, C_{2}$, and $C_{3}$ are device-specific coefficients. Prssi is the RSSI. Ptx is sent by the beacon to indicate its transmission strength. Ptx is typically defined to be the RSSI at a distance of one meter from the beacon using a reference Apple iPhone 5 device [5].

We used the default coefficients $\mathrm{C} 1, \mathrm{C} 2$, and $\mathrm{C} 3$ provided by the Android Beacon Library. For the best performance, these should be calculated separately for each phone model [5]. This was not done for this paper, however. Performing a device-specific calibration would improve distance calculation results to some extent.

The position estimate $(x, y)$ that minimizes the following is chosen:

$$
\sum_{\left(b_{x}, b_{y}, b_{d}\right) \in B}\left|\frac{\left[\left(x-b_{x}\right)^{2}+\left(y-b_{y}\right)^{2}\right]-b_{d}^{2}}{b_{d}^{n}}\right|
$$

Here $b_{x}$ and $b_{y}$ are the $x$ and $y$ coordinates of a beacon in the set $B$ of scanned beacons, and bd denotes the distance estimate according to equation 1 .

RSSI measurements are unreliable, and tend to become more unreliable with in-creasing distance. The term $b^{n}$ is used to assign larger weight to stronger beacons. We chose $\mathrm{n}=3$ in the measurements performed for this paper, as it was found to yield quite good results for our use case.

\section{Results}

Fig. 1 is a map, showing position estimates for the case where the phone is in the pocket, and the position is estimated to be at the beacon with the strongest signal. The black dots on the map are beacons. Some jitter has been added to the position estimates to prevent them from stacking on top of each other at the beacon location.

We can see that most of the position estimates are correct, i.e. the phone is estimated to be at a beacon in the space where the person was sitting. Some estimates, however, are assigned to a beacon outside the 
measurement location. For example, in some scans, the beacon outside the door of MR1 was estimated to be closer than the beacon inside the room. Similarly, in some cases the phone was estimated to be outside the rooms MR2 and SR3. The phone in SR1 was always correctly estimated, perhaps since there neigh-boring beacons were quite far away. The phone in SR2 was estimated to be at one beacon close to that room, since SR2 did not contain a beacon. Measurements in the coffee-room were quite spread out across the four beacons in the area, and were not always estimated to the physically closest beacon.

The map in Fig. 2 shows the position estimates calculated according to equation 2 . Here the position estimates tend to fall between the strongest heard beacons. This keeps the estimates mostly within the correct room for MR1 and SR3, while measurements in MR2 are "pulled" into MR1. The beacon in MR2 was placed quite close to the wall of MR1, however, which contributes to this. The measurements in SR2 are more accurate, while still not placed inside the correct room. Measurements in the coffee-room are estimated closer to the middle of the area in general.

Finally, Fig. 3 shows the estimates according to equation 2 when the phone was placed on a table. This improves the estimates significantly especially for measurements in the coffee-room, and also MR1. Estimates for SR3 are worse, however. Here we can see that the phone being in a pocket affects the results significantly. This is to be expected, however, since RF emissions at $2.4 \mathrm{GHz}$ are strongly attenuated by the human body.

In addition to the experiments in the Agora building, an initial test in the hospitals was done. This experiment is shown in Figure 4. A representative of one hospital was asked to walk between rooms of his choosing and make a note of the route. Two separate routes in the hospital were recorded with two different phones. The recorded path is shown in Fig. 4 using the estimation algorithm in equation 2. Relevant room numbers in the hospital have been added to the map. According to the notes provided by the hospital representative, the first route (Route 1 in Fig. 4) consisted of the following sequence of rooms: $5,4,3,2,1$. The second route was the following: $8,1,9,7,6$.

The position estimates of Fig. 4 correspond well to the noted routes. Some position estimates on the second route are incorrectly placed in rooms next to the route. This is one disadvantage of the current setup and estimation algorithms: position estimates while walking between rooms may not be reliable due to the rather long sampling time of half a minute, and also due to the relatively sparse placement of beacons in hallways outside of the rooms. These false readings could perhaps be minimized by for example filtering out estimates that remain stationary for only one half-minute period. 


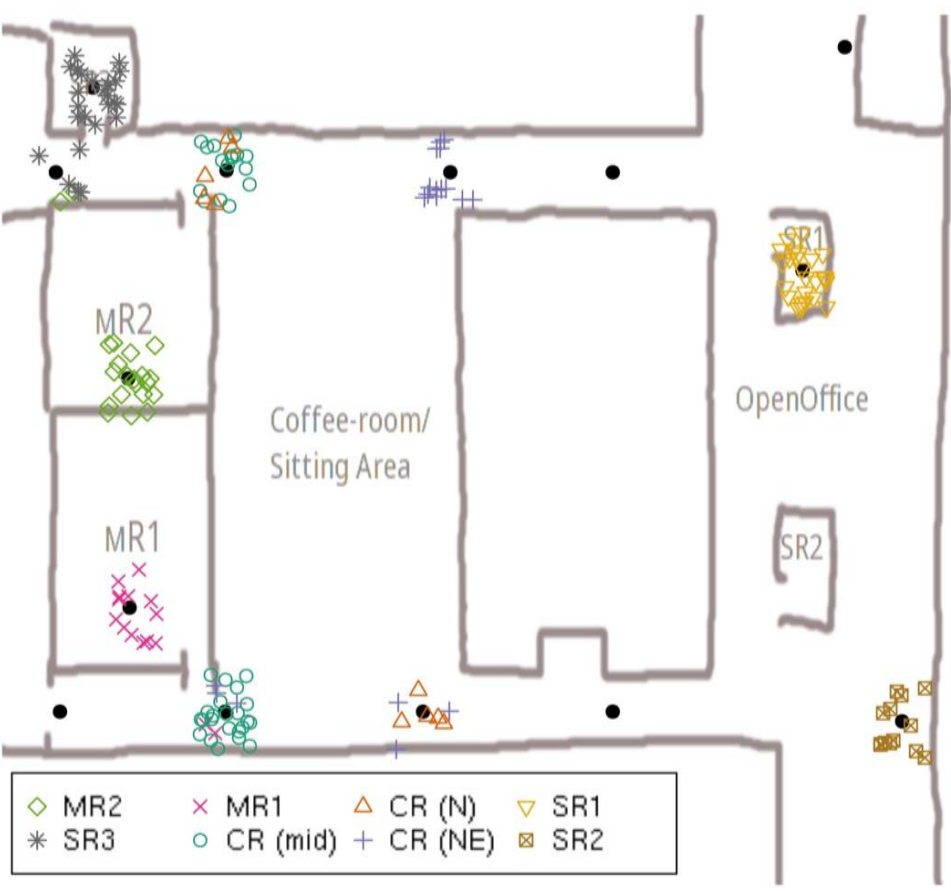

Figure 1. Closest beacon in Agora with the phone in a pocket.

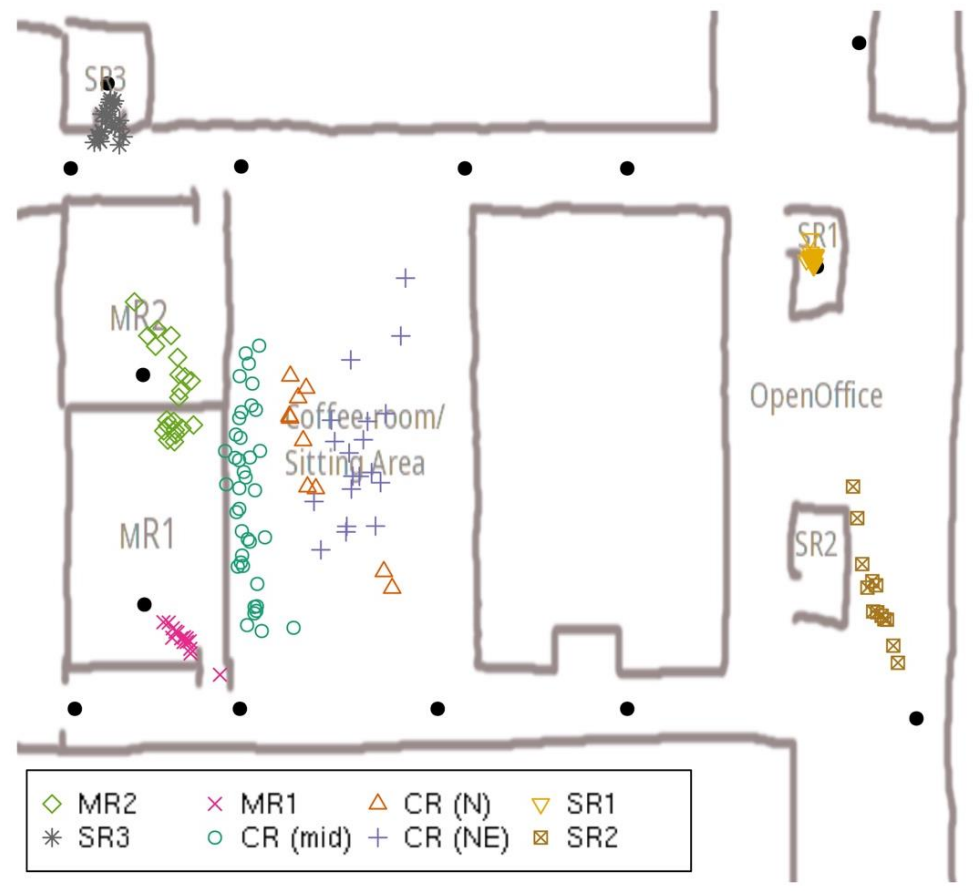

Figure 2. Position estimate in Agora with the phone in a pocket. 


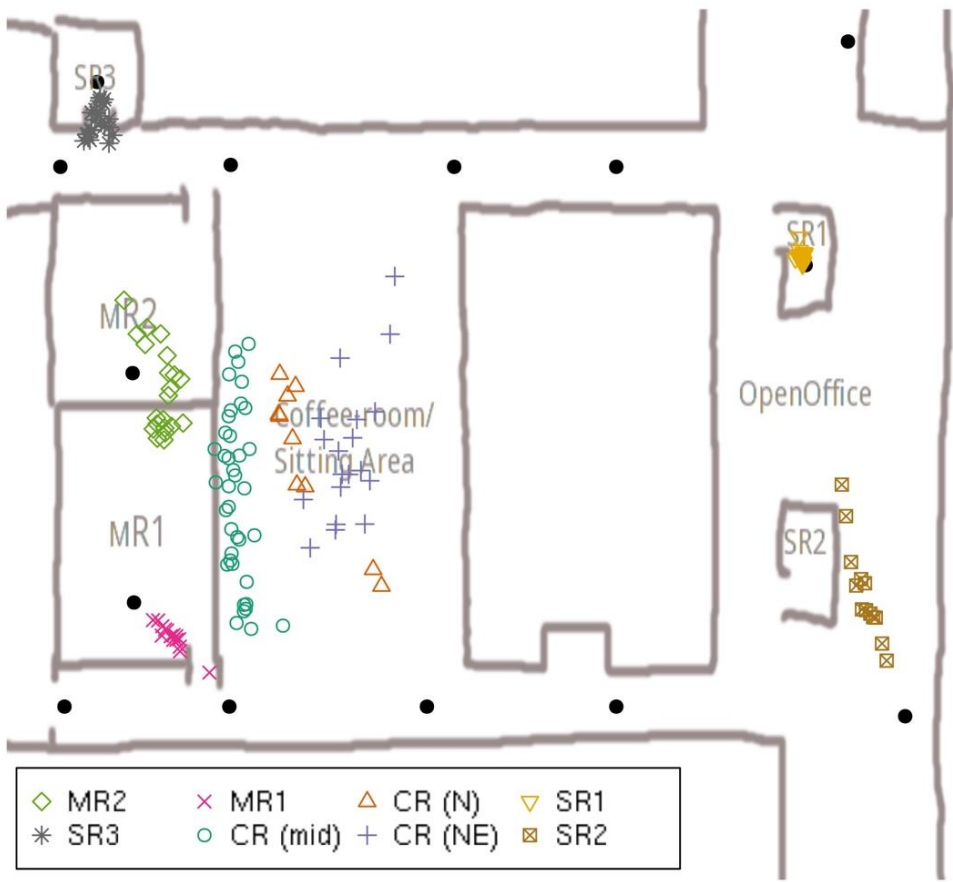

Figure 3. Position estimate in Agora with phone on table.

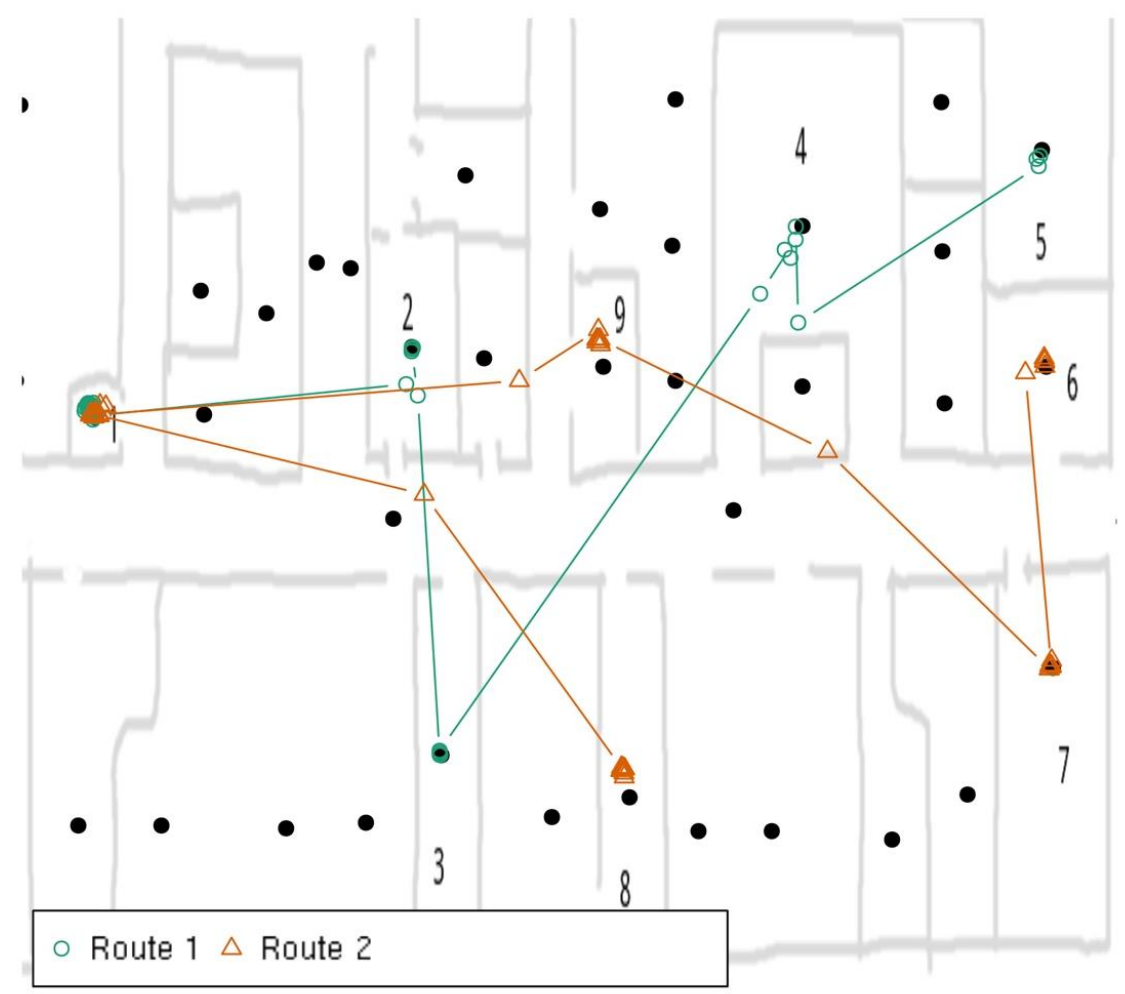

Figure 4. Two routes recorded in a hospital. 


\section{Discussion}

For this paper, we examined the accuracy of a simple indoor positioning system using Bluetooth beacons. Mobile phones record nearby beacons and their signal strengths in order to enable estimation of the phone's position. The system is suitable for short-term deployments due to its low cost and ease of installation. The system also requires minimal existing infrastructure in addition to the battery-powered beacons, and recording cellphones.

The experiments, which were performed in a university campus building showed that the system is able to provide approximately room-level accuracy. Especially, if the phone was carried in a pocket. The position estimates were erroneous at times, due to neighboring beacons' signals, which were stronger than the physically nearest beacon's signal.

Further field-trials performed in a hospital indicated that the system is fit-for-purpose. Some filtering of position estimates may be necessary during analysis, however.

\section{Ethical considerations}

Positioning systems may be used to track both humans and objects indoors and outdoors. This has a great potential in the health care setting. The tracking of scarce and expensive equipment reduces the risk of misplacements and supports the users to rapidly find needed equipment at the point of care. However, several ethical issues related to the privacy, the autonomy, the dignity and consent in the use of positioning systems with humans need to be addressed.

In general, tracking methods have been used with people who suffer from cognitive impairment, such as dementia [6]. These devices may reduce the time and effort required to find a missing person, increase the independence, autonomy and freedom of a person with middle- to moderate dementia, and reduce the caregivers level of stress [12]. The literature strongly suggests that these technologies should not be forced, and that the decision of using them should be made in formal meetings involving the individual concerned, the family members and the professionals involved in the care $[13,14]$. These issues should also be confronted during the product development and implementation phases [15].

Tracking technology has however the potential to support other users as well such as in tracking the activities of daily living, detecting falls, supporting visually impaired, and tracking people suffering from cardiovascular disease in case of a critical cardiac event [16]. However, the ethical aspects of use beyond the point of care in the elderly care setting has received little attention. The technology could for example be used to track professionals at work, but the motivation for doing this must be carefully considered and the consent of the professionals obtained. The technology could also be used to support researchers in a variety of areas, especially when assessing the impact of a new intervention. One such example is the tracking of professionals after an organizational change, and another the tracking of movement related to an exercise intervention.

Nonetheless, how and what data is collected, stored and used becomes extremely important when using tracking technology and privacy, autonomy, dignity and consent of the users must be assured. Whatever the reason for the interest in using positioning technology, the usage of such a system must always be carefully considered. The benefits of the use must always outweigh the possible harms and informed consent of all users must be obtained before implementation regardless of the purpose of the system implementation.

From an ethical point of view, the system in this study is opt-in: the beacons do not collect any data, and the system requires the user to actively run the phone application in order to be tracked.

\section{Conflict of interest}

All authors state no conflicts of interest. 


\section{References}

[1] Jiao J, Li F, Deng Z, Ma W. A Smartphone CameraBased Indoor Positioning Algorithm of Crowded Scenarios with the Assistance of Deep CNN. Sensors (Basel). 2017 Mar 28;17(4). pii: E704. https://doi.org/10.3390/s17040704

[2] Torres-Sospedra J, Jiménez AR, Knauth S, Moreira A Beer Y, Fetzer T, Ta VC, Montoliu R, Seco F, MendozaSilva GM, Belmonte O, Koukofikis A, Nicolau MJ, Costa A, Meneses F, Ebner F, Deinzer F, Vaufreydaz D, Dao TK, Castelli E. The Smartphone-Based Offline Indoor Location Competition at IPIN 2016: Analysis and Future Work. Sensors (Basel). 2017 Mar 10;17(3). pii: E557. https://doi.org/10.3390/s17030557

[3] Wang X, Jiang M, Guo Z, Hu N, Sun Z, Liu J. An Indoor Positioning Method for Smartphones Using Landmarks and PDR. Sensors (Basel). 2016 Dec 15;16(12). pii E2135. https://doi.org/10.3390/s16122135

[4] Belmonte-Fernández Ó, Puertas-Cabedo A, TorresSospedra J, Montoliu-Colás R, Trilles-Oliver S. An Indoor Positioning System Based on Wearables for AmbientAssisted Living. Sensors (Basel). 2016 Dec 25;17(1). pii: E36. https://doi.org/10.3390/s17010036

[5] Li F, Liu G, Liu J, Chen X, Ma X. 3D Tracking via Shoe Sensing. Sensors (Basel). 2016 Oct 28;16(11). pii: E1809. https://doi.org/10.3390/s16111809

[6] Wang Z, Yang Z, Dong T. A Review of Wearable Technologies for Elderly Care that Can Accurately Track Indoor Position, Recognize Physical Activities and Monitor Vital Signs in Real Time. Sensors (Basel). 2017 Feb 10;17(2). https://doi.org/10.3390/s17020341

[7] Gu Y, Lo A, Niemegeers I. A survey of indoor positioning systems for wireless personal networks. IEEE Communications Surveys Tutorials, 11(1):13-32, First 2009.

[8] Haverinen J, Kemppainen A. Global indoor selflocalization based on the ambient magnetic field. Robotics and Autonomous Systems 2009;57(10):10281035. 5th International Conference on Computational Intelligence, Robotics and Autonomous Systems (5th CIRAS). https://doi.org/10.1016/j.robot.2009.07.018
[9] Bluetooth SIG, Inc. Bluetooth low energy https://www.bluetooth.com/what- is-bluetoothtechnology/bluetooth-technology-basics/low-energy. Online; accessed 22.3.2016.

[10] Ji M, Kim J, Jeon J, Cho Y. Analysis of positioning accuracy corresponding to the number of ble beacons in indoor positioning system. In 2015 17th International Conference on Advanced Communication Technology (ICACT), pages 92-95, July 2015. https://doi.org/10.1109/icact.2015.7224764

[11] Young D. Fundamentals of beacon ranging. http://developer.radiusnetworks.com/2014/12/04/fun damentals-of-beacon- rang-ing.html. Blog post. Online; accessed 8.4.2016, Dec. 2014.

[12] Topfer LA. GPS Locator Devices for People With Dementia. CADTH Issues in Emerging Health Technologies. Ottawa (ON): Canadian Agency for Drugs and Technologies in Health; 2016-.147. 2016 Aug 31.

[13] Landau R, Werner S. Ethical aspects of using GPS for tracking people with demen-tia: recommendations for practice. Int Psychogeriatr. 2012 Mar;24(3):358-66. https://doi.org/10.1017/S1041610211001888

[14] White EB, Montgomery P. Electronic tracking for people with dementia: an ex-ploratory study of the ethical issues experienced by carers in making decisions about usage. Dementia (London). 2014;13(2):216-32. https://doi.org/10.1177/1471301212460445

[15] Yang YT, Kels CG. Does the Shoe Fit? Ethical, Legal, and Policy Considerations of Global Positioning System Shoes for Individuals with Alzheimer's Disease. J Am Geriatr Soc. $2016 \quad$ Aug;64(8):1708-15. https://doi.org/10.1111/jgs.14265

[16] Santoso F, Redmond SJ. Indoor location-aware medical systems for smart homecare and telehealth monitoring: state-of-the-art. Physiol Meas. 2015 Oct;36(10):R53-87. https://doi.org/10.1088/09673334/36/10/R53 